\title{
El crimen transnacional organizado como insurgencia no política: la experiencia Centroamérica
}

CArlos Murillo Zamora*

\begin{abstract}
Artículo recibido: 30 de agosto de 2015
\end{abstract}
Artículo aprobado: 3 de marzo de 2016

Doi: http://dx.doi.org/10.12804/desafios28.2.2016.05

Para citar este artículo: Murillo Zamora, C. (2016). El crimen transnacional organizado como insurgencia no política: la experiencia Centroamérica. Desafíos, 28(2): 177-211. Doi: http://dx.doi.org/10.12804/desafios28.2.2016.05

\section{Resumen}

El sistema internacional ha sufrido cambios profundos. Muchos de esos cambios han permitido visibilizar a actores no estatales; que en el pasado tenían espacios limitados por el control territorial y de flujos ejercido por los gobiernos. De igual manera en el caso de Centroamérica los conflictos político-militares de las décadas de 1970 y 1980 incrementaron la invisibilización de esos actores. Esos cambios generaron nuevos espacios de acción, que antes estaban copados por los gobiernos. A ello se suma el desarrollo del mundo virtual que favoreció la comunicación y el incremento de la presencia mediática de los actores no estatales. Esto posibilitó que distintos grupos criminales pudieran tener mayor influencia en las dinámicas sociales, económicas y politicas, repercutiendo en la cuestión de seguridad. Se agregan dos factores claves: un incremento de la violencia y una pérdida de legitimidad y credibilidad de las autoridades. De abi que cada vez aparezcan más espacios en donde la gestión pública se debilita y hasta desaparece; lo cual fue aprovechado por los grupos del crimen transnacional organizado. El desarrollo de estos grupos en el Istmo les ha permitido

\footnotetext{
* Profesor e investigador, Universidad de Costa Rica (UCR). Director del Doctorado en Gobierno y Políticas Públicas, UCR, San José, Costa Rica. Correo electrónico: carlos. murillozamora@ucr.ac.cr
} 
comenzar a asumir roles sociales e incluso a legitimarse en algunas comunidades y sectores sociales, al extremo que buscan infuenciar las estructuras de poder. Por ello es que se trata de "insurgencia no politica".

Palabras clave: crimen organizado, Centroamérica, Violencia, Insurgencia.

\title{
Transnational Organized Crime as Policy without Insurgency: The Experience of Central America
}

\begin{abstract}
The international system has undergone profound changes. Many of those changes have visible non-state actors; which in the past were limited by the territorial control and flow control exercised by governments. In addition, in the case of Central America's political-military conflicts of the 1970s and 1980s the invisibility of those actors was increased. These changes created new spaces for action that were controlled by the government. On the other hand, the virtual development favored communication and increased media presence of non-state actors. This enabled different groups to have more influence on social, economic and political dynamics, affecting the security issue. Two key factors are added: an increase in violence and a loss of legitimacy and credibility of the authorities. Hence, increasingly areas where governance is weakened and even disappeared; which is then exploited by transnational organized crime groups. The development of these groups in Central America has allowed them to begin to assume social roles and even be legitimized in some communities and social sectors. That is why it is "a non-political insurgency."
\end{abstract}

Keywords: Organized crime, Central America, violence, insurgency.

\section{O Crime Transnacional Organizado Como Insurgência Não Política: A Experiência Centro-americana}

\begin{abstract}
Resumo
O sistema internacional tem sofrido mudanças profundas. Muitas dessas mudanças têm permitido visibilizar a atores não estatais, que no passado tinham espaços limitados pelo controle territorial e de fluxos exercidos pelos governos. De igual maneira
\end{abstract}


no caso da Centro-América os conflitos politico-militares das décadas de 1970 e 1980 incrementaram a invisibilidade destes atores. Essas mudanças geraram novos espaços de ação, que antes estavam copados pelos governos. A isso soma-se o desenvolvimento do mundo virtual que favoreceu a comunicação e o incremento da presença mediática dos atores não estatais. Isto possibilitou que distintos grupos criminais puderam ter maior influência nas dinâmicas sociais, económicas e políticas, repercutindo na questão de segurança. Adicionam-se dois fatores chave: um incremento da violência e uma perda de legitimidade e credibilidade das autoridades. Dai que cada vez apareçam mais espaços onde a questão pública se debilita e até desaparece, o qual foi aproveitado pelos grupos do crime transnacional organizado. O desenvolvimento destes grupos no Istmo lhes tem permitido comecar a assumir papeis sociais e inclusive a legitimar-se em algumas comunidades e setores sociais, ao extremo que buscam influenciar as estruturas de poder. Por isso é que se trata de "insurgência não política".

Palavras-chave: Crime organizado, Centro-América, violência, insurgência.

El fin de la Guerra Fría no fue solo el cambio entre un orden internacional bipolar y la transición hacia otro multipolar o de otra naturaleza (el del siglo XXI), sino una variación en la estructura del sistema internacional, que puede ser comparado con el que tuvo lugar a inicios del siglo XIX o incluso el establecido por los Tratados de Paz de Westfalia en 1648. Esto porque significó una variación en la naturaleza y roles del Estado, en cuanto actor internacional; pues se generó una reconceptualización de las relaciones internacionales, que se manifiesta a través de una serie de eventos y procesos específicos, como el del crimen trasnacional organizado y el empoderamiento de otros actores no estatales nacionales e internacionales (legales o ilegales); teniendo repercusiones sobre el marco institucional estatal y el ejercicio del poder político.

Tales cambios han influenciado la naturaleza y dinámica en los ámbitos del quehacer político, económico, social, cultural, jurídico y estratégico. De esta forma algunos de los fenómenos dominantes en centurias anteriores, como el de la insurgencia política, también se han redimensionado. Por eso considero que se está frente a un mundo transformado, con una nueva arquitectura sistémica, generando la ruptura de las fronteras territoriales como se le concebían en el 
llamado "Estado westfaliano", al igual que el traslape de los espacios de acción que van de lo individual/ local hasta lo global, pasando por lo societal, estatal, regional e internacional. De esa forma es necesario observar nuevos eventos y procesos que resultan de las interacciones entre actores estatales y no estatales (tanto legítimos, como ilegítimos; lícitos e ilícitos) que operan en y a través de esos distintos niveles.

Lo anterior permite a autores como J. Rossenau $(1990,1997)$ hacer referencia a un mundo en el cual la turbulencia domina el cambio y la continuidad en la política mundial, con repercusiones en lo doméstico; al igual que a nuevas formas de gobernanza en ese mundo turbulento por los cambios en la frontera doméstico-exterior. Por ello este autor (Rossenau, 1990, p. 7-8) señala la necesidad de observar las dinámicas en los niveles micro y macro, teniendo en cuenta que la incertidumbre es una de las características del "nuevo mundo turbulento"; pues los eventos ocurren -gracias al desarrollo tecnológico- de forma prácticamente simultánea, como no se había observado en los tres siglos precedentes (Rossenau, 1990). ${ }^{1}$ Ello no debe inducir a olvidar que lo doméstico y lo internacional “...siempre han formado una red sin interrupciones y la necesidad de tratarlos como tal es urgente en tiempos de enorme transformación"; de ahí que "no podemos permitir que la frontera doméstico-exterior confunda nuestro entendimiento de los asuntos mundiales"2 (Rossenau, 1997, p. 4) y, lógicamente, de los internos.

De esa forma se debe comprender que cada vez hay menos procesos y fenómenos que se puedan considerar auténticamente domésticos; lo cual hace necesario pensar en temas y espacios transmésticos e intermésticos. Con ello se debe reconocer la profundidad de las

\footnotetext{
1 Rosenau (1990, p. 12-13) identifica como principales fuentes de cambio: i) la dinámica de la tecnología; ii) la emergencia de nuevos temas en la agenda global; iii) la limitada capacidad de los Estados y gobiernos para resolver los problemas; iv) frente al debilitamiento de los sistemas, los subsistemas han adquirido mayor coherencia y efectividad; y v) la retroalimentación de las consecuencias de los fenómenos ha incidido no solo en los tomadores de decisiones, sino también en el empoderamiento del individuo y colectividades no estatales.

2 Excepto que se indique lo contrario, en todos los casos la traducción de textos en otro idioma es responsabilidad del autor.
} 
variaciones; lo cual no quiere decir que se esté frente a un mundo diferente, sino transformado. En ese sentido resulta válido, a pesar de lo extenso, citar a Rosenau (1997) cuando acota:

[el] sistema internacional está menos al mando, pero aún es poderoso. Los Estados están cambiando, pero no desaparecerán. La soberanía estatal ha sido erosionada, pero aún es vigorosamente reivindicada. Los gobiernos son débiles, pero aún pueden magonear. En ciertas ocasiones los públicos son más demandantes, pero en otra son más maleables. Las fronteras aún mantienen a los intrusos fuera, pero también son más porosas. Los paisajes están dando paso a los espacios étnicos, mediáticos, ideológicos, tecnológicos y financieros, pero la territorialidad es aún una preocupación central para muchos pueblos (p. 4).

Entre los efectos de esos cambios, que me interesa abordar en este trabajo, están aquellos relacionados con la seguridad, la violencia -tanto política como general-, la insurgencia y el auge de los grupos del crimen organizado; porque están influenciando directamente el ejercicio del poder político en Estados frágiles, como los centroamericanos. Entendiendo poder no solo como un recurso que se tiene o posee -incluido el cómo apoderarse de éste- para ejercerlo sobre otros que carecen de este (Vallés, 2006, p. 31), propio de la concepción relacional del poder (Dahl, 1957), sino el para qué se quiere poseer y ejercer, y sobre todo a través de cuáles recursos se detenta. Al respecto hay que tener en cuenta que el poder político lo ejercen unos a través de la coacción física; mientras que otros recurren a la violencia para oponerse a las estructuras de poder, incluso utilizando las armas, como sucede con los movimientos insurgentes. Ello conduce a dos atributos del poder: legitimidad y legalidad.

Ahora bien, ante el incremento de la violencia y el surgimiento de amenazas a la integridad individual y colectiva, nacional e internacional, entre otros múltiples aspectos, que preocupan al ser humano, la cuestión de la seguridad se ha tornado en algo a lo cual prácticamente todas las personas se refieren. Sin embargo, se trata de una cuestión compleja, que es percibida de diferentes formas por 
individuos, gobiernos, corporaciones, especialistas académicos y distintas comunidades; haciendo referencia a cosas, personas, medios y fines, a eventos y fenómenos externos y situaciones sentimentales íntimas (cfr. McSweeney, 1999). Aunque históricamente lo relativo a seguridad se ha visto desde la perspectiva del Estado, considerando que este protege a su población de todo tipo de amenaza (cfr. Buzan, 1983).

La cuestión es que hoy tal premisa no resulta válida por distintas razones políticas, económicas, sociales y jurídicas; la mayoría de ellas derivadas de los cambios arriba mencionados. Pero también porque, como advierte B. Buzan (1983), durante el siglo XX fue un concepto subdesarrollado y limitado al enfoque estato-céntrico. Incluso, la violencia como fenómeno colectivo fue relacionada con movimientos sociales de naturaleza política, lo cual también está cambiando (Murillo, 2014). La otra violencia, interpersonal o a lo interno de pequeñas y medianas colectividades fue obviado por la Ciencia Política y Relaciones Internacionales. Así se acuñó el concepto de "violencia política" para hacer referencia a aquellos actos violentos con fines políticos (Della Porta, 2013, p. 2; Vallés, 2006, p. 35).

Lo anterior conduce a indagar acerca del papel que desempeñan los grupos del crimen organizado (ya sea transnacional o no) en la dinámica política de los países centroamericanos, pues su acción ha influido directamente en el ejercicio del poder político. Lo cual se ha visto favorecido por el debilitamiento y fragilidad institucional de los Estados, que deja espacios sin atender, abriendo posibilidades para que esos grupos satisfagan las necesidades de sectores sociales, que los legitiman. Por lo que planteo como pregunta orientadora ¿en qué grado la acción e influencia del crimen organizado -nacional y transnacional- está convirtiendo el fenómeno en un tipo de insurgencia no política, redefiniendo las relaciones políticas y el ejercicio del poder político en Centroamérica?

La premisa que guía este documento es que los grupos del crimen organizado $(\mathrm{CO})$, sobre todo aquellos del crimen transnacional 
organizado (CTO), como los carteles del narcotráfico y pandillas tipo maras, constituyen un forma de insurgencia que busca modificar el aparato estatal para generar espacios que le garanticen sus beneficios económicos y sociales, pero sin que necesariamente les interese detentar en forma directa el poder político; por ende esas agrupaciones tienen fines e intereses políticos.

En este artículo hago primero referencia breve a la cuestión de seguridad y la creciente fragilidad del Estado. En una segunda sección exploro lo relativo a la insurgencia y su evolución; para en tercer lugar analizar lo relativo al fenómeno del crimen organizado y las tendencias que lo caracterizan. En una cuarta sección considero la actual situación centroamericana, antes de formular algunas consideraciones generales.

\section{Seguridad y Estado: una perspectiva general}

De acuerdo con B. McSweeney (1999, p. 3) es necesario tener en cuenta la cualidad relacional de la seguridad respecto a la inseguridad, no con relación a distribución de capacidades materiales, amenazas y vulnerabilidades. El problema es que el predominio del Estado como el referente de todos los procesos, domésticos e internacionales, influyó en la noción de seguridad como el resultado de la posesión de esos recursos materiales (poder duro) para repeler, disuadir o eliminar las amenazas externas; puesto que las internas eran responsabilidad del aparato estatal. El poder era concebido como una cuestión de posesión de recursos y por ende de estatus quo, en función del "interés nacional", lo que condujo a tener como punto de partida la tesis de la seguridad nacional (cfr. Wolfers, 1967). Esta se convierte en la preocupación de todo gobierno, en la medida que el sistema internacional se caracterizaba por la anarquía y la lucha de todos contra todos, lo que hace -desde la perspectiva de Hobbes-que los Estados se centren en la lucha por la seguridad (Wolfers, 1967, pp.10-11). Incluso la identidad estatal se construye en función de la preparación para confrontar las amenazas, convirtiéndose la inseguridad en la constante (McSweeney, 1999, p. 5). 
De esa forma seguridad se concibe a partir de la presunción teórica de la primacía del Estado, la escasa relevancia de las subunidades y el predominio de los recursos materiales, dejando al individuo como subordinado del Estado (McSweeney, 1999). Con ello surgió la doctrina de la seguridad nacional, que durante el tercer cuarto del siglo XX considera que la insurgencia y otras formas de violencia política eran producto de las amenazas provenientes del sistema internacional. Mientras que la inseguridad individual resultaba de la delincuencia común y por tanto no era un problema de seguridad para el Estado. La cuestión, se pregunta B. Buzan (1983, p. 52), es “...cómo aplicar un concepto como seguridad a una cosa tan efímera como una idea o un conjunto de ideas", por lo que "...el problema de seguridad es mitigado por la inherente dificultad de inducir al cambio”.

Conforme aparecieron nuevos enfoques en las ciencias políticas durante las décadas de 1970 y 1980, por una parte, y el surgimiento de teorías sobre las relaciones internacionales, como la Interdependencia Compleja, y sobre las dimensiones de la política (cfr. Vallés, 2006, cap. 3), lo cual implicaba un cambio en la dinámica estatal, comenzó a reconocerse la urgencia de ensanchar el concepto de seguridad (McSweeney, 1999, cap. 3). Se pasa de seguridad frente a amenazas militares a una orientada a la sobrevivencia a través de distintas dimensiones (Castle, 1997, p. 1).

Lo anterior condujo a que se plantearon dos formas principales de análisis de la seguridad: una es la estato-céntrica, basada en la concepción tradicional; y otra en un sentido más amplio que comprende no solo el Estado sino que abarca una extensa agenda, incluyendo los sectores económico, ambiental y societal (Buzan, Wæver \& de Wilde, 1998). Complementado con la necesidad de analizar la cuestión de seguridad reconociendo las interacciones en y entre niveles de análisis, que comprenden el sistema internacional, los subsistemas internacionales, las unidades (actores estatales y no estatales), las subunidades y los individuos (Buzan et al, 1998). Esto permite profundizar en aspectos propios de cada región y no solo abordar la seguridad entre lo doméstico y lo externo; por lo que se deben tener en cuenta los "complejos de seguridad regional" (cfr. Murillo, 2012); pues tras el fin de la Gue- 
rra Fría (GF) y el colapso de la bipolaridad los escenarios regionales adquirieron mayor relevancia (Buzan et al, 1998, p. 9).

Entonces se estableció un escenario en el que las citadas "amenazas no tradicionales" que fueron visibilizadas, condujeron a nuevos problemas y nuevas inseguridades, así muchos asuntos fueron "seguridizados", llegando a la agenda pública y convirtiéndose en un asunto cotidiano en todos los ámbitos. Por consiguiente, A. Castle (1997, p. 5) advierte que la seguridad hoy se caracteriza por:

i. el mantenimiento de los valores centrales de una sociedad;

ii. la libertad de la población de esa sociedad desde las amenazas graves a las existenciales;

iii. el mantenimiento por las autoridades constituida del control sobre la legitimidad del uso de la fuerza; $y$

iv. el mantenimiento de las autoridades constituidas del control sobre el territorio nacional delimitado.

La seguridad llegó de lleno a disciplinas como Ciencias Políticas y Relaciones Internacionales, ensanchándose no solo su agenda, sino los escenarios y actores relacionados. Se comenzó a pensar tanto en seguridad pública como privada, individual y colectiva, el rol de la tecnología, la idea de la seguridad humana, al mismo tiempo que la seguridad ontológica. Esto adquirió mayor relevancia tras los atentados del 11 de setiembre de 2011, lo cual mostró que la seguridad también tiene que ver con valores, creencias y otros factores que en el pasado habían sido invisibilizados; como también que la revolución en las tecnologías de información y comunicación, complementadas con el transporte, habían empoderado al individuo y grupos (lícitos e ilícitos) frente al control estatal sobre el espacio territorial. De esta forma aparece el concepto de ciberseguridad, para contrarrestar los ataques cibernéticos, tanto a los sistemas informáticos como a las acciones cotidianas de las autoridades. ${ }^{3}$

\footnotetext{
3 Sobre este tema véase, entre muchas publicaciones, el trabajo de L. Larkin (2004) los significados de la seguridad en el contexto de la información.
} 
Ahora bien, no hay que perder de vista que la seguridad tiene un fuerte componente de percepción, por lo que tanto seguridad como inseguridad son subjetivas; de ahí que la "cultura de seguridad" permite una mayor tolerancia o aceptación de ciertos delitos, pero se opone a otros, no necesariamente por su grado de violencia, sino por fundamentarse en "narrativas locales explicativas del delito, de sus causas y soluciones" (Kessler, 2008, p. 107).

Hay que tener en cuenta que ante el miedo y el temor la gente prefiere aliarse con el que se muestra poderoso y competente. Así, por ejemplo, surgen los narco-referentes, como los narco-corridos, las narco-novelas y el líder narco como un líder que resuelve algunos problemas, o el caso de Pablo Escobar como dirigente político. En El Salvador hay gente que prefiere encerrarse en su ámbito privado para evitar ser objeto de ataques. Hay que tener en cuenta las construcciones identitarias y los mensajes, sobre todo de los medios de comunicación que inciden en ello.

Tales cambios coincidieron con un debilitamiento de las instituciones en un gran número de Estados, lo que convirtió a muchos de estos en Estados frágiles (ineficaces, débiles, fracasando y fallidos, que poseen desafíos para la gobernabilidad interna y la gobernanza regional y global [Schneckener, 2006, p. 23]), generando mayores espacios para la gestión privada individual y colectiva, junto con una creciente pérdida de control sobre el territorio y sobre los flujos transfronterizos. Para D. Álvarez (2014) es evidente que:

[...] Son muchos los Estados de la región que han perdido el monopolio del poder coercitivo, mientras asistimos a una proliferación de actores con intereses privados, atomizados, que ejercen de facto el control sobre diversas áreas del territorio y sus poblaciones... dicha pérdida de control y legitimidad está erosionando el pacto social, minando la autonomía del Estado y, en ciertos casos extremos, amenaza incluso con su desintegración (p. 34).

Por consiguiente, se está frente a una interacción muy compleja en la que el actor estatal enfrenta viejos y nuevos retos y desafíos do- 
mésticos, que se suman a amenazas tradicionales y no tradicionales a la seguridad nacional. La ciudadanía se ha empoderado, por lo que exige mayor democracia y mejores oportunidades para garantizar su bienestar individual y colectivo; pero ante los crecientes costos para realizar de forma apropiada las actividades que le corresponde a los gobiernos, incrementa la condición de inseguridad. Esto, como ya lo mencioné, empodera a actores no estatales, quienes aprovechan los espacios dejados por el Estado; pero al mismo tiempo provoca mayores brechas entre las distintas comunidades, tanto en lo urbano como en lo rural. Por ejemplo, "[el] Estado se debilita y el cumplimiento de las normas públicas es reemplazado por el uso de mecanismos privados de justicia y de seguridad", lo cual no significa que la ciudadanía renuncie a resolver los conflictos, sino que esta defensa "ahora se hace por fuera de las instituciones del Estado y normalmente valiéndose del uso de la violencia” (Álvarez, 2014, p. 37).

Lo anterior conduce a problemas de gobernanza y gobernabilidad, lo cual aumenta la fragilidad del Estado y amenaza a la democracia, que se supone la mejor forma para implementar políticas que generen equidad e incrementan las acciones de toda la población, contribuyendo a la seguridad humana y al desarrollo individual y colectivo (Bertranou \& Calderon, 2008, pp. 15-16). Ello genera un círculo en el cual la democracia -entendida como régimen institucional y como forma de vida- contribuye a y se beneficia de la seguridad humana -como sustituto de la seguridad nacional- lo cual conduce al desarrollo humano; la cuestión es que no se puede perder de vista que “...la gobernabilidad democrática será más compleja en la medida que no esté garantizada la seguridad humana, sobre todo en países como los latinoamericanos con importantes rezagos en pobreza y desigualdad" (Bertranou \& Calderon, 2008, p. 16).

Entonces, se debe reconocer que "...la violencia y la inseguridad son, además, un obstáculo para la consolidación de la gobernabilidad democrática", lo cual ha conducido a que:

[...] las dificultades del sector de seguridad para reducir los niveles del delito han incentivado la adopción de políticas o prácticas por 
fuera del Estado de Derecho, como las experiencias de acciones de "limpieza social" que se llevan adelante en ciertas ciudades (Álvarez \& Manzotti, 2008, p. 32). ${ }^{4}$

Ello incrementa el debilitamiento de y la pérdida de credibilidad en las instituciones estatales, estableciéndose un círculo vicioso; que se torna más difícil de romper porque las sociedades modernas dependen en alto grado de la función policial y estos cuerpos no han estado dentro de las prioridades de los gobiernos en los países latinoamericanos, en donde la prioridad son las fuerzas armadas. Es decir, a mayor violencia e inseguridad, mayor descrédito de las instituciones estatales para atender las demandas de la población, que busca otras vías para satisfacer sus necesidades.

En este análisis no se puede obviar la perspectiva de género, incluso se puede afirmar, considerando el caso centroamericano, ${ }^{5}$ que en muchos casos la violencia tiene rostro femenino, pues la razón o tasa de sexo es un factor que incide en los cambios societales y está relacionado con la violencia y la criminalidad (Hudson \& Leidl, 2015). Hay una tendencia a que los jóvenes prefieren órdenes basados en la fuerza física, considerando que poseen una ventaja; de ahí que Hudson \& Leidl (2015) se cuestionen el ¿por qué las alteraciones en la tasa de sexo afectan la estabilidad societal? Ellas consideran que los jóvenes adultos tienden a vincularse más en conductas criminales y autodestructivas, sobre todo cuando no tienen una relación sentimental o están casados.

\footnotetext{
4 En este documento no analizo la relación entre crimen organizado y violencia, pues constituye otra variable del problema que requiere un estudio específico. Además, hasta la fecha los estudios no logran establecer una correlación entre violencia/conflicto y pobreza. 5 V. Hudson y P. Leidl (2015, p. 111) señalan que Guatemala es un Estado caracterizado por una alta tasa de asesinatos y el femicidio está entre las más altas del mundo, pero también la mitad de la población infantil (menores de 5 años) tienen desnutrición crónica; se suma una de las más bajas tasas impositiva. Para ellas "Guatemala es solo otro ejemplo de cómo el síndrome de la toma de alquiler depredadora por el poder puede destruir el futuro de un país -sus niños-. La alta mortalidad y morbilidad infantil son manifestaciones primarias del tipo de nacionalismo masculino maligno...".
} 
A la perspectiva de género hay que agregar otros aspectos sobre el cómo se estudian las sociedades, culturas y económicas, lo cual es determinante para explicar y entender los fenómenos; pues como lo advirtió E. Wolf (citado van Schendel, 2005, p. 38) el mundo no puede ser entendido como "bolas de billar socioculturales, moviéndose en una mesa de billar global'. La cuestión es que, como lo señalé desde la perspectiva del Estado, la visión dominante, de acuerdo con W. van Schendel (2005) se caracteriza porque:

Las ciencias sociales llegaron a existir conforme los Estados territoriales modernos estaban surgiendo a un protagonismo sin precedentes en el mundo. No es extraño que los científicos sociales se plantaran en lo intimidante del Estado: ante sus ojos casi toda la humanidad y toda la superficie de la tierra fue colocado bajo su dominio. Un sistema de Estados históricamente único basado en el poder y la soberanía territorializada proporcionada por los científicos sociales con un marco para conceptualizar sociedades, culturas, naciones, historias, y economías. De hecho, la estructura territorial del sistema interestatal moderno llegó a ser ampliamente aceptada como un modelo general de organización socioespacial, y la mayoría de los científicos sociales analizaron la vida social como si esta estaba siendo jugada en las unidades geográficas auto-cerradas (p. 38).

Así surge un "territorialismo metodológico", un "estatismo empotrado" y una "trampa territorial", que hoy enfrenta nuevas formas de conectividad, provocando que cada vez menos el Estado territorial "contenga" sociedades (van Schendel, 2005, p. 39) y menos naciones. A ello se suma, como ya mencioné, el problema de los cambios en las fronteras estatales y los crecientes flujos trasfronterizos, sobre todo ilegales, según la consideración de los gobiernos. Ahora esos flujos abarcan migrantes, individuos, inversiones, bienes y servicios, adquiriendo una magnitud que no hace muchas décadas era impensable. Sin embargo, solo ha sido estudiado en términos cuantitativos y en menor grado respecto a sus efectos socioculturales y políticos sobre las sociedades penetradas por esos flujos o a través de las cuales esos desplazamientos se dirigen a otros Estados. Para el citado 
autor (Schendel, 2005, p. 49) “...estudiar los flujos ilegales en las zonas fronterizas provee con entendimientos especiales sobre cómo la territorialidad y la transnacionalidad son negociados en prácticas diarias y cómo la gente "escala" el mundo en que viven".

En definitiva, es necesario no solo tratar de explicar y entender la amplia naturaleza de la seguridad, la actual estructura y funciones de los actores estatales y no estatales, los flujos transfronterizos y los problemas de la gobernabilidad y gobernanza para lograr un acercamiento a los cambios que están ocurriendo, puesto que como anota U. Schneckener (2006, p. 25) “...la relación entre Estados frágiles y actores no estatales armados y sus consecuencias para la gobernanza" están convirtiéndose de factores determinantes de lo político, la política y las políticas, al mismo tiempo que reconocer que esos actores no estatales armados usan la violencia para alcanzar sus objetivos y no se integran en instituciones estatales formalizadas (Schneckener, 2006, p. 25; cfr. Swyngedouw, 2014); aunque sí constituyen agentes con intereses políticos y no solo económicos, a lo cual me refiero en la siguiente sección.

\section{Insurgencia: su naturaleza y actual caracterización}

Históricamente la insurgencia, ${ }^{6}$ entendida como “. ...actores no estatales que buscan transformar su sociedad políticamente en alguna forma significativa y que también usan la violencia estratégicamente para alcanzar un fin político" (Dallas-Feeney, 2013, p. 14; cfr. Salehyan, 2011, p. 11), pudiendo actuar en forma clandestina o movilizando a las masas como apoyo al movimiento insurgente, se ha concebido como un fenómeno doméstico. Para Estados Unidos (Marines, 2012, p. 1) la insurgencia es “...una lucha político-militar prolongada dirigida a subvertir o desplazar la legitimidad del gobierno constituido u ocupar el poder y controlar, total o parcialmente los recursos de un territorio a través del uso de las fuerzas militares irregulares y las organizaciones políticas ilegales". Pero también, de acuerdo con B.

\footnotetext{
6 Esta sección tiene, sobre todo en su primera parte, como base el artículo sobre insurgencia no política ya citado (Murillo, 2014).
} 
O’Neil (citado por Hammes, 2006, p, 18) se puede concebir como "...una lucha entre un grupo no gobernante y las autoridades en la cual el grupo no gobernante usa conscientemente recursos políticos y violencia para destruir, reformular o sostener las bases de uno más aspectos de la política" (énfasis en original).

Como tal el grupo insurgente busca debilitar el "control y legitimidad del gobierno, mientras incrementa el control y legitimidad insurgente" (Dallas-Feeney, 2013, p. 15). Es decir (Marines, 2012, p. 2) la insurgencia aspira a:

i. minar la capacidad del gobierno para proveer seguridad y servicios a la población;

ii. obtener el apoyo activo o pasivo de la población;

iii. provocar al gobierno a cometer abusos con la población; y

iv. minar el apoyo internacional al gobierno y ganar reconocimiento $\mathrm{o}$ asistencia

Ello hace que la legitimidad se convierta en un factor clave de este tipo de organizaciones, por cuanto como "forma de lógica relacional" es parte del espectro de control social en la medida en que los actores participantes -subordinados- consideran válida y creíble la gestión que realizan los grupos que ejercen el poder o buscan replantear la relación de poder (Dallas-Feeney, 2013, p. 17). Estos tratan de ganar simpatía entre la población, de forma que su lucha sea validada en términos de generar una mejor condición de vida, ante lo cual hay disposición a someterse a la coerción que ejerce quien detenta el poder (Bach, 1996, p. 38).

Relacionado con ello está el factor de "mentes y corazones", que opera tanto para los movimientos insurgentes que buscan el apoyo popular, como para los gobiernos que aspiran a legitimar sus operaciones contrainsurgentes. Esto hace necesario considerar el rol de la violencia y la búsqueda del apoyo en situaciones de insurgencia (Mahoney, 2011); lo cual solo menciono, pero sin profundizar, por razones de espacio, a pesar de la relevancia que tiene esta cuestión 
para comprender el éxito o fracaso de los movimientos insurgentes y particularmente con el caso de las proto-insurgencias.

Otra consideración que es necesario tener en cuenta sobre la insurgencia es que se le ha vinculado a concepciones ideológicas particulares, sobre todo al leninismo, maoísmo, castrismo e islamismo fundamentalista, por lo que su concepción y caracterización está en función de lo que explican y proponen esas ideologías; de ahí que “...sin estas ideologías las teorías de la motivación y la acción insurgente colapsan como una casa de cartas" (Bach, 1996, p. 34). Entonces la insurgencia se convirtió en un fenómeno estrictamente basado en la desafiliación política de quienes la impulsan, respecto al orden establecido. Ello tiene sentido cuando la observación se hace en el contexto de las "guerras revolucionarias"; pero no cuando el contexto está en función de otros actores e intereses.

No se puede perder de vista el carácter cambiante y la naturaleza dinámica de los grupos insurgentes. Estos buscan “....cambiar el foco del conflicto lejos de los dominios en donde el Estado u otra estructura de poder es particularmente fuerte" y también procura dominar en donde "...la moral y otras características sicológicas importan más que el poder tangible", caracterizándose por su sobrevivencia, su fortalecimiento y el debilitamiento de las estructuras estatales de poder (Metz, 2012, p. 80).

Por otra parte, es necesario aludir al apoyo a los movimientos insurgentes, porque este resulta, según un estudio de RAND (Davis et al, 2012, p. 4) de un "gran caldero de actividades", en el cual se pueden observar no solo procesos políticos, sino también de seguridad, sociales y económicos, interconectados. Es decir, el contexto y entorno en que se produce la insurgencia debe ser considerada en su observación, no pudiendo simplemente extrapolarse los resultados de una experiencia a otros escenarios; puesto que existen dinámicas y singularidades que repercuten de forma particular en los procesos sociales como la insurgencia, tanto desde una perspectiva cualitativa como cuantitativa de la observación que se haga (Davis et al, 2012, p. 1). De ahí la necesidad de entender por qué las insurgencias no florecen con la 
misma dinámica, ni presentan las mismas características, ni tampoco lo hacen de forma instantánea o automática cuando en un escenario se presentan coyunturas similares a otras situaciones. Entonces resulta conveniente diferenciar entre insurgencias y proto-insurgencias. Estas corresponden con “...pequeños grupos violentos que buscan ganar el tamaño necesario para alcanzar más efectivamente sus metas y usar recursos tales como la movilización política y la guerra de guerrillas también como el terrorismo" (Byman, 2012, p. 1). La cuestión es que grupos proto-insurgentes que no logran evolucionar, pueden permanecer en esa fase preliminar de proto-insurgencia, buscando generar los espacios necesarios para su sobrevivencia.

Entonces se trata de una confrontación asimétrica, que se diferencia de los conflictos entre gobiernos y grandes movimientos insurgentes revolucionarios, por lo cual deben ser entendidos como lo que son: proto-insurgencias.

Por consiguiente, la situación de la insurgencia -y de la citada protoinsurgencia- varía al generarse fenómenos transnacionales, porque los Estados tienen ventaja para usar la fuerza cuando operan en su propio territorio, pero su poder está limitado cuando se trata de traspasar sus fronteras (Salehyan, 2011). Esto ocurre cuando los gobiernos enfrentan a grupos rebeldes transnacionales, definidos como “....grupos opositores armados cuyas operaciones no están confinadas al territorio geográfico del Estado-nación que desafían” (Salehyan, 2011, p. 15).

Por otra parte hay que considerar que los grupos y redes sociales transnacionales muestran un dinamismo mucho mayor y capacidad de movilización a través de los distintos espacios de las comunidades políticas (polities), en claro contraste con los Estados, que no son tan móviles y flexibles (Salehyan, 2011, p.34). Ello aplica tanto para grupos con objetivos estrictamente políticos, como para otros movimientos, incluidos los ilícitos. Esto alcanza niveles críticos en el caso de los Estados frágiles, pues carecen de recursos suficientes para tener un control real y efectivo sobre la totalidad del territorio (Salehyan, 2011, p. 41), lo que genera mayores espacios para que los actores rebeldes desarrollen sus actividades y adquieran mayor legitimidad. 
Cabe señalar que S. Metz (2012, pp. 1-2) identifica múltiples modelos de insurgencia, en términos de cómo cada grupo insurgente alcanza sus objetivos. Hay tres destacados: i) proto-Estado adoptado por los movimientos maoístas; ii) el reemplazo del Estado a través de su destrucción; y iii) la insurgencia no política. Sobre este tercer tipo señala:

Más que buscar reemplazar al Estado, este tipo de insurgencia quiere debilitar el Estado lo suficiente para estar libre de su control. Normalmente, es el intento de los insurgentes practicar alguna forma de actividad criminal organizada. Como en todo lado el crimen organizado, estos insurgentes buscan más que activo apoyo popular que pasividad. Aunque los métodos de tales insurgentes son similares a los insurgentes enfocados políticamente (Metz, 2012, p. 2).

Sin embargo, J. Garzón (2012) advierte que no se puede igualar al $\mathrm{CO}$ con grupos insurgentes o terroristas; sobre todo porque el CO tiene motivaciones económicas, no políticas, el objetivo no es tomar el poder, sino reformularlo para crear condiciones favorables para sus actividades. Acerca de esta tesis me refiero más adelante.

Autores como E. Swyngedouw (2014) identifican otros movimientos insurgentes, como Indignados y "Democracia Real Ya". Esto es importante porque durante un buen tiempo lo político fue dejado en segundo plano, desconociendo el rol que tiene en la construcción y evolución de la sociedad.

En resumen, lo descrito en esta sección evidencia que la insurgencia es un fenómeno complejo, que tiene múltiples expresiones, a diferencia de lo que ocurría en siglos anteriores.

\section{Crimen organizado: un fenómeno en crecimiento}

El CO e incluso su variante el CTO no son fenómenos recientes, a lo largo de la historia de la humanidad ha habido grupos que coordinan para realizar actividades consideras ilícitas. Sin embargo, no existe una definición de consenso a pesar de su importancia y la preocupación 
expresada por numerosos actores. Así la resolución 56/120 de la Asamblea General de ONU, adoptada el 19 de diciembre de 2001, se refirió al "impacto del crimen transnacional organizado sobre la estabilidad política, social y económica y el desarrollo de las sociedades". Agrega "la lucha contra la delincuencia organizada trasnacional es responsabilidad común de toda la comunidad internacional y requiere cooperación bilateral y multilateral". Por ello, A. Orlova y J. Moore (2005, p. 269) advierten que tales definiciones se deben construir teniendo en cuenta las convenciones anti-crimen organizado, al mismo tiempo que de convenciones internacionales sectoriales. Las primeras constituyen la sombrilla y las segunda el complejo de edificios en la "construcción incremental de marcos legales comprehensivos".

La Convención de Palermo o Convención de las Naciones Unidas contra la Delincuencia Organizada Transnacional, aprobada por la AGNU en noviembre de 2000 y abierta a su firma en la Conferencia de Palermo de diciembre de ese año, utiliza los siguientes conceptos (art. 2):

a) Por "grupo delictivo organizado" se entenderá un grupo estructurado de tres o más personas que exista durante cierto tiempo y que actúe concertadamente con el propósito de cometer uno o más delitos graves o delitos tipificados con arreglo a la presente Convención con miras a obtener, directa o indirectamente, un beneficio económico u otro beneficio de orden material;

$[\ldots]$

c) Por "grupo estructurado" se entenderá un grupo no formado fortuitamente para la comisión inmediata de un delito y en el que no necesariamente se haya asignado a sus miembros funciones formalmente definidas ni haya continuidad en la condición de miembro o exista una estructura desarrollada (2002, s.p.).

A. Orlova y J. Moore (2005, pp. 282-83) consideran que tales definiciones son contradictorias, porque hacen referencia a grupos estructurados jerárquicos y grupos no jerárquicos con una escasa 
definición de roles de sus miembros, resultado de abarcar a una diversa gama de organizaciones, pues la definición alude a "uno o más crímenes graves como uno de los elementos claves que caracterizan a tal grupo", cuando advierten que diversos autores -tales como M. Woodwiss, M. Beare y R. Naylor-coinciden en que "lo que distingue a las entidades criminales organizadas de otros grupos es la actividad criminal sistémica y regular".

A. Mazzitelli (2007) considera que los grupos del CO operan a partir de principios racionales, minimizando el riesgo y maximizando las ganancias. Por ello se ajustan a las condiciones específicas del entorno en donde operan, lo cual hace necesario diferenciar entre los grupos de las distintas regiones geográficas (por ejemplo, para el caso africano cfr. Mazzitelli, 2007, p. 1085).

En el caso del CTO hoy se considera como un asunto de "baja política” en el ámbito internacional (Castle, 1997, p. 4), porque como señala P. Williams (citado por Castle, 1997, p. 4) esas organizaciones amenazan la seguridad nacional e internacional, al tiempo que adquieren condiciones que les hace resistentes a los esfuerzos para contenerlas, afectarlas o destruirlas. Por eso L. Shelley (citada Castle, 1997, p. 4) considera que "...el fracaso para desarrollar políticas internacionales viables y coordinadas frente a la siempre creciente criminalidad transnacional puede minar el Estado-nación en el siglo XXI", por lo cual J. McFarlane y K. McLellan consideran que el CTO se está constituyendo en una grave amenaza para la seguridad y estabilidad nacional e internacional (citados Castle, 1997, p. 4).

El CO opera a lo largo de varios ejes. Por ejemplo la Conferencia Ministerial sobre CTO, celebrada en Nápoles en 1994, lo caracterizó a partir de:

i. grupo organizado para cometer crímenes;

ii. posee vínculos jerárquicos o relaciones personales que permite a los líderes controlar el grupo;

iii. la violencia, intimidación y corrupción son usadas para obtener ganancias o controlar territorios o mercados; 
iv. el lavado de ilícitos sirve para intensificar la actividad criminal e infiltrar la economía legítima;

v. potencial para la expansión dentro de cualesquiera nuevas actividades y más allá de las fronteras nacionales; y

vi. cooperación con otros grupos criminales trasnacionales organizados.

Ahora bien, hay que tener en cuenta que existen crímenes que son transnacionales, pero no organizados y crímenes organizados pero no transnacionales (Castle, 1997). Además, no toda actividad delictiva, aunque sea realizada por dos o más personas se puede considerar como organizada. De ahí que exista una tendencia a considerar como CO y CTO solo aquellas actividades que tienen una estructura organizacional y responden a un interés económico. Es decir, grupos que tienen una motivación económica, no ideológica ni de búsqueda del poder, aunque si necesita influir en la política; que se caracterizan por una estructura jerárquica, con una autoridad que planifica y controla con un gran profesionalismo, una fuerte disciplina interna y posee como un aliado clave la corrupción; aprovecha la tecnología; mantiene una permanente diversificación; y constituye un sector dinámico, generador de riqueza y de empleo (PNUD, 2009, p. 102).

En este sentido se pronuncia J. Rivera (2011) cuando plantea en forma extensa que:

el crimen organizado, que existe para hacer dinero, es en esencia una empresa económica, que normalmente se ha diversificado en lo local y en lo transnacional. El crimen organizado no tiene ideología ni principios políticos, lo que la diferencia de otras organizaciones, por ejemplo, las organizaciones terroristas, aunque unas y otras pueden compartir métodos y tácticas de violencia. Cuando el crimen organizado entra en el ámbito político, por ejemplo como sucede en Rusia o Taiwán, lo hace con la exclusiva finalidad de favorecer aún más sus intereses. El crimen organizado por lo tanto medra y se sostiene a través de la oferta de servicios de los que existe una fuerte demanda pero que básicamente son ilegales o escasos, como ejemplo, algunos de los 
bienes ilícitos son, la adopción de bebés, las drogas, tráfico de animales y plantas exóticas, el tráfico de armas ilegales, tráfico de órganos humanos, el tráfico de niños y los objetos robados, y entre los servicios ilegales están el juego, el lavado de dinero y el sexo, la pornografía infantil (p. 6).

Tales afirmaciones caen en la tendencia a obviar lo político o a dejarlo en segundo plano, como si los distintos actores no estatales y comunidades en general no fueran, ante todo, agentes políticos (cfr. Swyngedouw, 2014). No porque el CO y el CTO carezcan de una ideología, como se concebía en la centuria anterior, no son actores políticos. Incluso el mismo J. Rivera (2011, p. 17-18) termina reconociendo la influencia e incidencia del CO sobre el Estado, pues identifica como efectos de la acción de esos grupos:

i. población demanda del Estado más protección y compromiso, lo cual es una obligación constitucional;

ii. incremento del presupuesto de seguridad pública;

iii. pérdida de confianza en los funcionarios públicos y erosión del Estado de derecho;

iv. penetración del sistema político por el financiamiento de campañas políticas para mantener relaciones de poder $;^{7} \mathrm{y}$

v. efectos negativos sobre la democracia, la población responsabiliza a la democracia de la inseguridad.

Sin duda lo anterior demuestra la naturaleza política del CO y su complejidad (PNUD, 2009, p. 34). Por ello es necesario tener en cuenta la relación agente-estructura para comprender la naturaleza del CTO (cfr. Edwards \& Gill, 2002). Sobre todo cuando la dinámica que esas organizaciones muestran permiten considerar que en América Latina se está frente a una "rebelión de las redes criminales" (Garzón, 2012, p. 1), puesto que:

\footnotetext{
Sobre esto cabe recordar el concepto de "sistemas políticos penetrados" que acuña J. Rosenau (2006, p. 183): “...es uno en el cual lo no miembros de una sociedad nacional participan directa y autoritariamente, a través de acciones tomadas en conjunto con miembros de la sociedad, en la distribución de sus valores o la movilización de apoyo en nombre de sus objetivos".
} 
i. hay disputas entre facciones del CTO, que compiten por obtener un mayor control;

ii. represalias contra el Estado;

iii. búsqueda de actores "legales" para involucrarse en actividades criminales;

iv. densidad criminal y acumulación social de violencia e ilegalidad; $\mathrm{y}$

v. reemplazo de capos por brokers.

De ahí que se debe adoptar una perspectiva apropiada para explicar y comprender la naturaleza y dinámica del $\mathrm{CO}$, que supere las concepciones restringidas del pasado, como advierten T. Pankratz y H. Matiasek (2012) cuando anotan:

[...] Una mirada al panorama nos lleva a reconocer quel el CO no puede seguir siendo visto como un fenomeno criminal abnormal, pero con definicion clara, en ciertos paises y regiones. En su lugar, debemos estar concientes de que el $\mathrm{CO}$ es un fenomeno que toca todas las areas y actores de la sociedad, incluyendo aquellos dedicados a combatir y controlar el delincuencia organizada. Incluso los Estados desarrollados, durante mucho tiempo caracterizados como inmunes al CO debido a su tejido social intacto, hoy están confrontados con el desafío del CO (p. 42).

Existe un significativo consenso que el CO logra mayores avances en países en donde hay desajustes sociales y un mayor grado de anomia (PNUD, 2009, p. 99, también cfr. Alda, 2015). La coincidencia entre este fenómeno y la creciente fragilidad de los Estados ha hecho, como señalan F. Reinares y C. Resa (1997, p. 3) que haya pasado, en una década, de ser considerado un problema propio de unos pocos países y regiones, a “.... ser uno de los factores básicos cuando se definen las amenazas a la seguridad nacional en general y la gobernanza democrática en particular”. Por ello especialistas como L. Shelley (1995, p. 463) advierten que se trata de una amenaza a la integridad de los países, que resulta de "...la complejidad de estas organizaciones y sus actividades, su penetración global y la amenaza que posee a la democracia y legitimidad del desarrollo económico", y se ve favorecido por 
un Estado débil (Alda, 2015). De ahí la necesidad de -para comprender este fenómeno- reconocer dos variables: complejidad y continuidad; pues ello permite diferenciar entre el crimen común y el organizado. El primero es más simple (más difuso) y espontáneo que el segundo (Bailey \& Ortega, 2010, p. 3). Por supuesto a mayor complejidad y continuidad mayor violencia.

De acuerdo con A. Castle (1997, p. 10) las principales actividades que caracterizan las acciones del CTO incluyen:

i. tráfico de drogas y sustancias sicotrópicas;

ii. tráfico de armas convencionales;

iii. tráfico de materiales nucleares;

iv. tráfico de mujeres y menores;

v. contrabando de extranjeros ilegales;

vi. robo de autos en gran escala;

vii. tráfico de órganos;

viii. lavado de dinero;

ix. evasión fiscal; y

x. corrupción.

Cabe anotar, solo como referencia, que de acuerdo con Y. Zabyelina (2013) el CTO ha tenido una gran mutación, adoptando formas sofisticadas que involucra la vinculación entre actores criminales y élites políticas, incluidos agentes diplomáticos. Estos facilitan o participan en el tráfico ilegal, abusando de su inmunidad y privilegios.

Sin embargo, es necesario tener en cuenta que hay una tendencia, a todas luces errónea, a pensar ciertos fenómenos como situaciones desvinculadas entre sí, aun cuando son procesos. En el caso de los grupos criminales hay que tener en cuenta que operan en un continium y su ubicación a lo largo de eje varía según su evolución. Por ejemplo, no se puede considerar a las pandillas como un fenómeno desconectado totalmente de las maras; estas evolucionaron a partir de aquellas. Además, es necesario reconocer la existencia de traslapes entre distintas manifestaciones del crimen organizado, que han 
generado alianzas y esquemas de colaboración, como se observa en el caso centroamericano.

\section{Centroamérica: un escenario del crimen organizado}

En Centroamérica se identifican 23 principales "mercados subterráneos", con dimensión territorial local, nacional o transnacional: cables telefónicos robados; tapas de alcantarilla robadas; luminarias y cables eléctricos robados; energía eléctrica, agua y otros servicios domiciliarios de contrabando; teléfonos celulares robados; accesorios de vehículos robados; repuestos y partes automotrices robadas; combustibles y lubricantes; semovientes; violación a la ley de derechos de autor; contrabando; robo, exportación y comercialización ilegal de bienes culturales; tráfico de animales protegidos; comercialización ilícita de maderas preciosas; fraudes o estafas con tarjetas de crédito; robo de vehículos; tráfico de armas de fuego; tráfico de inmigrantes; trata de personas; tráfico de órganos; tráfico interno de drogas; tráfico internacional de drogas; y lavado de dinero y otros activos (PNUD, 2009, p. 101). Tal descripción refleja la magnitud del negocio del $\mathrm{CO}$ en el Istmo.

Sin embargo, lo anterior debe contextualizarse, reconociendo que en Centroamérica se produjo un cambio profundo tras el fin de los conflictos armados, que coincidió con cambios en el orden internacional y en la arquitectura sistémica, al mismo tiempo una transformación en las estructuras sociales producto de algunas dinámicas globalizadoras, sobre todo de naturaleza socio-cultural. Mientras que los gobiernos estaban preocupados en la transición hacia la democracia y las políticas económicas, por lo que no percibieron y menos tomaron conciencia de la naturaleza, dinámica y profundidad de los cambios domésticos, regionales, internacionales y globales, junto con el debilitamiento de la frontera entre lo doméstico y lo externo. En ese contexto comenzaron a consolidarse, por una parte, y a aparecer, por otra, nuevas formas de violencia y criminalidad. Sin olvidar que en el caso de Centroamérica la mayoría de los Estados no habían completado su construcción (state-building) o incluso carecían de ella (caso de Honduras). Así la década de 1990 constituyó un punto de convergencia de múltiples 
dinámicas, procesos y eventos de muy diversa naturaleza. Cuando se hacían esfuerzos para intentar comprender esa situación, bajo la tesis de un mundo unipolar, llega el 9-11 que introduce otro giro en el escenario global. Entonces sin completarse la transición hacia los distintos escenarios - político, económico, geoestratégico- que enfrentaba cada país centroamericano, en particular, y el Istmo, en general, se produce un turning-point que replanteó el sistema internacional y las relaciones entre actores estatales y no estatales.

Como señala L. Chinchilla (2002, p. 169-70) se debe tener en cuenta que en esta región se produjeron cambios en las décadas de 1990 y 2000 en materia de seguridad y de roles de los cuerpos de defensa y seguridad (ejércitos y policías). Así se adopta el Tratado Marco de Seguridad Democrática y se establece el Instituto Superior de Estudios Policiales de Centroamérica, orientados a la parte doctrinaria y a la redefinición del marco conceptual, normativo e institucional, por una parte; y los esfuerzos técnico-operativos tendientes a mejorar la eficiencia de los cuerpos nacionales a través de iniciativas regionales, por otra. En este segundo caso aparecieron entidades como Asociación de Jefes de Policía de Centroamérica, Tratado de Asistencia Legal Mutua en Asuntos Penales, Comisión Centroamericana Permanente para la Erradicación de la Producción, Tráfico y Consumo Ilícito de Estupefacientes y Sustancias Psicotrópicas (CCP), Tratado Centroamericano sobre Recuperación y Devolución de Vehículos Hurtados, Robados/Apropiados o Retenidos Ilícita o Indebidamente, y el Convenio Centroamericano para la Prevención y la Represión de los Delitos de Lavado de Dinero y de Activos, relacionados con el Tráfico Ilícito de Drogas y Delitos Conexos.

Entonces, lo que se puede observar es la convergencia de tres fenómenos en el caso centroamericano: el fin de la GF y la desintegración del bloque soviético; el auge del CO; y los cambios producto del final de los conflictos armados, el proceso de democratización y del aumento de la dinámica integracionista.

G. Molina (2009, p. 3) señala que el clima de inseguridad que enfrenta el Istmo es generado tanto por la violencia (asesinatos, robos, asaltos) 
como por el pobre desempeño de las instituciones relacionadas con la seguridad, lo cual incrementa los sentimientos de desconfianza de la ciudadanía, ello “....se traduce en la importancia que los gobiernos y sus instituciones sean percibidos como incompetentes en el área de seguridad pública, ya que la pérdida de confianza en las instituciones, supone un elemento importante que afecta el funcionamiento de la democracia” (Molina, 2009, p. 3).

Esa situación se agrava por la magnitud del problema de la violencia, que C. Moser y A. Winton (2002, p. 2) caracterizan por:

i. múltiple complejidad de la violencia diaria;

ii. factores causales apoyando la multiplicad de la violencia;

iii. costos y consecuencias de la violencia;

iv. marco operacional para intervenciones en el Istmo; y

v. violencia en curso relacionada con las intervenciones.

Por consiguiente es necesario construir una perspectiva general que incorpore los indicadores demográficos, socioeconómicos, de pobreza y exclusión social, junto con los de violencia. Lo cual no hago aquí por razones de espacio.

Ello es importante porque la violencia permea la realidad cotidiana, particularmente en las comunidades pobres, traslapando ámbitos y niveles para dar lugar a complejas expresiones de violencia en múltiples capas de la sociedad (Moser \& Winton, 2002).

Como señala C. Realuyo (2013) Centroamérica ha sido una de las regiones más afectadas por el CTO, producto de los niveles de violencia que acompañan el tráfico de drogas, armas y personas; por lo que esta autora (Realuyo, 2013, p. 4) advierte que estos grupos “... están transformando los países centroamericanos de simples puntos de tránsito a construcción de fuentes, producción, transporte y consumo de su contrabando de narcóticos, armas y personas".

Y si además se tiene en cuenta, como anotan Reinares y Resa (1997, p. 19) el CO “...puede interferir en la cultura política de un país 
dado... en el conjunto de valores y actividades relacionadas con el entendimiento de la actividad política por los individuos y colectividades que construyen la sociedad", por lo que influyen en los ámbitos social y físico, distorsionando la frontera entre lo legal e ilegal, cambiando las lealtades hacia las instituciones y repercutiendo en el funcionamiento del sistema.

Por ello Solís \& Floglesong (2009, p. 11) señalan que hay una creciente preocupación en la región porque de alguna forma “....el crimen organizado parodiará la democracia, erosionará el Estado de derecho, entorpecerá la estructura social y comprometerá la seguridad pública. El crimen organizado no pasará sin notarse en la región".

Como señala un reporte de World Bank (2011) Centroamérica enfrenta una oleada de violencia comparable con la del periodo de guerras civiles. Ello se manifiesta en diversas formas y con diferentes consecuencias. Las primeras se resumen en los costos directos del crimen y de su combate, los costos por la caída en la productividad y la desmotivación de la inversión extranjera; la situación de las familias víctimas de la violencia; y la deslegitimación y pérdida de credibilidad del aparato estatal, entre muchas otras.

\section{Algunas consideraciones finales}

Los cambios que han ocurrido en las pasadas décadas, tanto en el sistema internacional, como en el doméstico, han generado múltiples variaciones en las relaciones políticas entre actores individuales y colectivos, estatales y no estatales, al extremo que es necesario comprender que muchos procesos y fenómenos sean redimensionado y enfrentan nuevas dinámicas, modificándose en mayor o menor grado su naturaleza. Sin embargo, desde la perspectiva de la mayoría de las ciencias sociales particularmente de la ciencia política y de relaciones internacionales persisten muchos conceptos y criterios, parámetros y metodologías basadas en la ontología y epistemología del siglo XX, sobre todo continua predominando la visión estato-céntrica, lo cual dificulta una apropiada observación de los eventos y actores empoderados del mundo transformado del siglo XXI. 
Otro aspecto a considerar es la relación entre agente y estructura, que se produce en un contexto cultural, político, económico, social, jurídico y estratégico modificado; lo cual también influye en las interacciones entre los diferentes y numerosos agentes. A ello se suma el debilitamiento de las fronteras estatales y del control gubernamental sobre el espacio territorial, lo cual permite -en buena medida gracias a la revolución de las TIC- mayores flujos transnacionales de personas, bienes y servicios.

Lo anterior ha contribuido a empoderar a actores que en el pasado estaban invisibilizados, sobre todo a individuos y grupos, sean estos ilegales e ilegítimos, lo cual incrementa los retos y desafíos del aparato estatal, que se ve limitado para satisfacer las crecientes demandas de esos diversos actores, tales como las pandillas y carteles del narcotráfico. Entonces, conforme las instituciones estatales muestran menos capacidad de acción y gestión, los otros buscan otros referentes y vías para alcanzar sus metas y satisfacer sus necesidades. Ello genera un círculo vicioso que repercute directamente en la diada seguridadinseguridad, sobre todo en su dimensión subjetiva.

En ese complejo contexto uno de los fenómenos que más se ha visibilizado y empoderado son los grupos del CO. Muchos de estos, a diferencia de sus versiones anteriores, recurren a su visibilización y a la búsqueda de legitimidad. Así, su mayor presencia y difusión junto con la magnitud de sus actividades, principalmente de naturaleza económica y social, provocan una mayor pérdida de credibilidad del Estado, que se muestra incapaz de combatir sin recurrir al uso de la fuerza. Sin embargo, esto genera más violencia, que se suma a aquella que conlleva las acciones del CO. En resumen la violencia se torna exponencial y los grupos criminales no dejan de aumentar y sus ganancias de multiplicarse.

Ahora bien, es válido señalar que el CO tiene ante todo un propósito económico, pero la dimensión de sus operaciones y la proliferación de estas organizaciones incrementan sus interacciones políticas y sociales. Esto no las convierte en agentes políticos; sin embargo, si adquieren un rol en lo político, por lo que inciden en la política y en 
las políticas. Además hay que tener en cuenta que el $\mathrm{CO}$ requiere de ciertas garantías para operar y de espacios de maniobra. Esto solo lo genera el Estado, por lo que la relación con las élites políticas y gobernantes es un factor clave, que se logra a través del establecimiento de redes y es facilitado por la corrupción. Es decir, sin el Estado los grupos criminales no podrían existir; porque a estos los benefician un estado con una débil institucionalidad. Por lo que en los casos de Guatemala y Honduras, y en menor grado El Salvador, los carteles del narcotráfico han penetrado las estructuras de poder político y las pandillas - particularmente en su versión de maras- tienen un gran control de los espacios comunitarios. Esto se hizo evidente durante las elecciones presidenciales salvadoreñas de 2014, en las que las maras tuvieron un rol en el triunfo del actual Presidente Sánchez Cerén.

Por supuesto que al CO no le interesa ejercer directamente el poder político, pues tendrían que asumir responsabilidades que desviarían su atención de sus negocios. Ello no quiere decir, repito, que no tengan objetivos políticos, que son perseguidos a través de sus vínculos con líderes y contactos dentro de las burocracias o con la participación directa en puestos de decisión de algunos miembros del CO -el caso paradigmático fue Pablo Escobar en Colombia-. Sin duda esto no significa ejercer o pretender el poder político.

Ahora bien, si se entiende, como indiqué en la sección sobre insurgencia, que esta es un actor no estatal que trata de transformar la sociedad en alguna forma, recurriendo a la violencia y subvirtiendo o desplazando la legitimidad del gobierno, entonces cuando se trata del CO se está frente a un tipo particular que no busca tomar el poder y ejercerlo, pero sí influencia lo político y las estructuras estatales. Por eso es que considero que se trata de una insurgencia no política. Los grupos del CO necesitan mantener la presión sobre el Estado para garantizar que sus actividades continúen. Sin esto el CO no podría subsistir.

El problema que tiene el Estado es que combate la criminalidad con mano dura, lo cual como señale, incrementa la espiral de violencia. Pero si busca otras vías, legitimaría a esos grupos como interlocutores, 
y no necesariamente estos desaparecerán. Fue lo que sucedió en El Salvador con el acuerdo entre el gobierno y las maras. En las últimas décadas Centroamérica ha sido una especie de laboratorio en lo político, económico, social y estratégico. Lo cual se ve favorecido por factores de convergencia y divergencia en todos los ámbitos. Así mismo por las dinámicas fronterizas y la conformación de las distintas regiones dentro de los países.

En el caso de Centroamérica es evidente el carácter de insurgencia no política que tiene el CO, sobre todo en los grupos de narcotráfico, maras y mafia. En general, constituyen redes con vínculos domésticos, centroamericanos y regionales. Esto no quiere decir que tengan una relevancia similar en todos los países.

Honduras constituye el mayor ejemplo de penetración en la estructura política de los carteles del narcotráfico y las maras. Mientras que en Guatemala el predominio son los grupos de narcotraficantes, tanto nacionales como transnacionales, con una participación de las maras. En estos dos países el debilitamiento del estado es evidente. El Salvador muestra un comportamiento diferente porque no existen pruebas de una gran influencia del narcotráfico, pero sí de las maras que fueron legitimadas con el pacto, resultado de una negociación propia de gobierno y movimientos insurgentes.

Costa Rica y Panamá constituyen casos en donde si bien la violencia es menor, existen grupos del CO dedicados al tráfico de drogas y contrabando de personas, bienes y servicios. Ambos países tiene pandillas, pero no se ha comprobado una alta penetración de maras. Lo que sí es evidente la limitada capacidad institucional para combatir el CO.

Mientras que Nicaragua muestra una situación particular con una aparente presencia de grupos del CO. Sin embargo, son pocos los estudios que explican las razones de esta tendencia. Esto no quiere decir que no existan organizaciones vinculadas al narcotráfico, sobre todo en la costa del caribe. Por consiguiente, en este país se requiere mayor análisis para identificar algunos factores que permitan comprender el fenómeno. 


\section{Referencias}

Alda, S. (2015). ¿El crimen organizado prefiere Estados débiles o fallidos? Esglobal. 24 de junio de 2015. Recuperado de: http://www.esglobal. org/elcrimenorganizadoprefiere estadosdebilesofallidos/

Álvarez, A. \& G. Manzotti. (2008). El estado de la seguridad en América Latina. En A. Álvarez, J. Bertranou, \& D. Fernández (Eds.), Estado, democracia y seguridad ciudadana. Aportes para el debate (pp. 31-55). Buenos Aires: Programa de Naciones Unidas para el Desarrollo.

Álvarez, D. (2014). Desafíos en la agenda regional de seguridad ciudadana y criminalidad transnacional organizada. Opera, 15, 33-54.

Bach,W. (1996). Youth Gangs and Insurgency Theory: Perceptions of Legitimacy and Coercion amongst a Disaffiliated Populace. (Thesis of Master of Arts). The Pacific Lutheran University: Tacoma WA.

Bailey, J. \& D. Ortega. (2010). Interactive Dynamics "Common" and "Organized" Crime in Latin American Cities: Concepts and Hypothesis. Paper prepared for the Conference "Common Crime and Organized Crime in Latin American Cities: Commonalities and Differences, May 19, 2010. Washington DC; Woodrow Wilson International Center for Scholars.

Bertranou, J. \& F. Calderon. (2008). Introducción. En A. Álvarez, J. Bertranou, \& D. Fernández (Eds.), Estado, democracia y seguridad ciudadana. Aportespara el debate (pp. 11-29). Buenos Aires: Programa de Naciones Unidas para el Desarrollo.

Buzan, B. (1983). People, States and Fear. Sussex, GB: Whesheaf Books Ltd. Buzan, B., Wæver, O. \& J. de Wilde. (1998). Security. A New Framework for Analysis. Boulder, CO: Lynne Rienner Publishers.

Byman, D. (2012). Understanding Proto-Insurgencies. Santa Monica, CA: RAND National Defense Research Institute.

Castle, A. (1997). Transnational Organized Crime and International Security. CIR Working Paper 19. Vancouver: Institute of International Relations, The University of British Columbia.

Chinchilla, L. (2002). Estabilidad social y seguridad ciudadana en Centroamérica. En F. Carrión (Ed.), Seguridad ciudadana, iespejismo o realidad? (pp. 167-187). Quito: FLACSO.

Dahl, R. (1957). The Concept of Power. Behavioral Science, 2, 201-215. 
Dallas-Feeney, C. (2013). The Social Fitness of Insurgencies: The Organizational Payoff for Legitimated Power. (Dissertation for the degree of Doctor of Philosophy) The George Washington University, Washington DC.

Davis, P., Larson, E.V., Haldeman, Z., Oguz, M. \& Rana, Y. (2012). Understanding and Influencing Public Support for Insurgency and Terrorism. Santa Monica, CA: RAND National Defense Research Institute.

Della Porta, D. (2013). Clandestine Political Violence. Cambridge, UK: Cambridge University Press.

Edwards, A. \& P. Gill. (2002). The politics of 'transnational organized crime': discourse, reflexivity and the narration of 'threat'. British Journal of Politics and International Relations, 4(2), 245-270.

Garzón, J. (2012). The Rebellion of Criminal Networks: Organized Crime in Latin America and the Dynamics of Change. Washington DC: Woodrow Wilson Center Update on the Americas.

Hammes, T. (2006). Countering Evolved Insurgent Networks, Military Review, July-August, 18-26.

Hudson, V. \& Leidl, P. (2015). The Hillary Doctrine. Sex and American Foreign Policy. New York: Columbia University Press.

Kessler, G. (2008). Inseguridad subjetiva: nuevo campo de investigación y de políticas públicas. En A. Álvarez, J. Bertranou \& D. Fernández (Eds.), Estado, democracia y seguridad ciudadana. Aportes para el debate (pp. 107142). Buenos Aires: Programa de Naciones Unidas para el Desarrollo.

Larkin, L. (2004). Meanings of Security: A Constructivist Inquiry into the Context of Information Security Policy Development Post 9/11. (Doctoral Dissertation). Virginia Commonwealth University, Richmond, VA.

Mahoney, C. (2011). Hearts and Minds or Blood and Guts? Strategy, Terrorism, and the Growth of Proto-Insurgencies. (Dissertation for the degree of Doctor of Philosophy). University of California, Los Angeles CA.

Marines. (2012). Guide to the Analysis of Insurgence. Washington DC; United States Marine Corps. Recuperado de http://www.mccdc.marines.mil/ Portals/172/Docs/ SWCIWID/COIN/Doctrine/Guide\%20to\%20 the $\% 20$ Analysis $\% 20$ of $\% 20$ Counterinsurgency.pdf

Mazzitelli, A. (2007). Transnational organized crime in West Africa: the additional challenge. International Affairs, 83(6), 1071-1090.

McSweeney, B. (1999). Security, Identity and Interests. A Sociology of International Relations. Cambridge, UK: Cambridge University Press. 
Metz, S. (2012). The Internet, New Media, and the Evolution of Insurgency. Parameters. Autum: 80-90.

Molina, G. 2009. Una evaluación al estado de las instituciones de seguridad pública: una comparación entre el Triángulo Norte de C.A. y Nicaragua. ¿Alguna diferencia? Buenos Aires; CLACSO. Recuperado de http://bibliotecavirtual.clacso.org.ar/clacso/ becas/20120511111830/ molina.pdf

Moser, C. \& A. Winton. (2002). Violence in the Central American Region: Towards an Integrated Framework for Violence Reduction. London: Overseas Development Institute.

Murillo, C. (2012). Regiones, seguridad y Estados frágiles. El caso de Centroamérica. Security and Defense Studies Review, 13, 237-266.

Murillo, C. (2014). Insurgencia no política: la experiencia en Centroamérica y México. Una aproximación preliminar. Trabajo presentado en el curso de Terrorismo y Contrainsurgencia, William J. Perry Center for Hemispheric Defense Studies, National Defense University; Washington DC. Inédito.

Orlova, A. \& J. Moore. (2005). "Umbrellas" or "Building Blocks"?: Defining International Terrorism and Transnational Organized Crime in International Law. Houston Journal of International Law, 27(2), 267-310.

Pankratz, T. \& H. Matiasek. (2012). Understanding Transnational Organised Crime. A Constructivist Approach towards a Growing Phenomenon. SLAL Journal-Journal for Police Science and Practice, 2, 41-50.

Programa de las Naciones Unidas para el Desarrollo (PNUD). (2009). Abrir espacios para la seguridad ciudadana y el desarrollo humano. Informe sobre Desarrollo Humano para América Central, IDHAC, 2009-2010. Colombia; Programa de las Naciones Unidas para el Desarrollo.

Realuyo, C. (2013). Harnessing Economic Development to Counter Trasnational Organized Crime in Central America. Miami, FL: Center For Hemispheric Policy, University of Miami.

Reinares, F. \& C. Resa. (1997). Transnational organized crime as an increasing threat to the national security of democratic regimes: assessign political impacts and evaluating state responses. NATO Research Fellowship Program 1997-1999. Recuperado de http://www.nato.int/acad/fellow/97-99/reinares.pdf revisado 7 de julio de 2015 .

Rivera, J. (2011). El crimen organizado. Guatemala; Instituto de Estudios en Seguridad. Recuperado de http://www.galileo.edu/ies/files/2011/04/ EL_CRIMEN_ORGANIZADO-IES.pdf 
Rosenau, J. (1990). Turbulence in World Politics. A Theory of Change and Continuity. Princeton, NJ: Princeton University Press.

Rosenau, J. (1997). Along the Domestic-Foreign Frontier: Exploring Governance in a Turbulent World. Cambridge, UK: Cambridge University Press.

Salehyan, I. (2011). Rebels without Borders. Transnational Insurgencies in World Politics. Ithaca: Cornell University Press.

Schneckener, U. (2006). Fragile Statehood, Armed Non-State Actors and Security Governance. En A. Bryden \& M. Caparini (Eds.), Private Actors and Security Governance (pp. 23-41). Zurich: Geneva Centre for the Democratic Control of Armed Forces (DCAF).

Shelley, L. (1995). Transnational Organized Crime: An Inminent Threat to the Nation-State? Journal of International Affairs, 48(2): 463-489.

Solís, L. \& T. Foglesong. (2009). Organized crime and its impact on democratic societies. En F. Rojas, \& L. Solís (Eds.), Organized Crime in Latin America and the Carribbean: Summary of Articles (pp. 11-20). San José: FLACSO.

Swyngedouw, E. (2014). Recuperado de http://www.cades.be/ckfinder/ userfiles/files/SwyngedouwSpaceAndPolityESFinalJuly2013.pdf

Vallés, J. (2006). Ciencia Política. Una Introducción (5 Ed.) Barcelona: Editorial Ariel.

van Schendel, W. (2005). Spaces of Engagement. How Borderlands, Illegal Flows, and Territorial States Interlock. En W. van Schendel \& I. Abraham (Eds.), Illict Flows and Criminal Things: States, Borders, and the Other Side of Globalization (pp. 38-68). Bloomington IN: Indiana University Press.

World Bank. (2011). Crime and Violence in Central America: A Development Challenge. Washington DC: World Bank. Recuperado de: www.worldbank. org/lac revisado 22 de junio de 2015.

Wolfers, A. (1967). Discord and Collaboration. Essays on International Politics. Baltimore: The Johns Hopkins Press.

Zabyelina, Y. (2013). The untouchables: transnational organized crime behind diplomatic privileges and immunities. Trends Organ Crim, 16, 343-357. doi: 10.1007/s12117-012-9184-y 
